\title{
Black hole formation from collapsing dust fluid in a background of dark energy
}

\author{
Rong-Gen Cai ${ }^{1}$ and Anzhong Wang ${ }^{2}$ \\ 1 Institute of Theoretical Physics, Chinese Academy of Sciences, P.O. Box 2735, Beijing 100080, China \\ ${ }^{2}$ CASPER, Physics Department, Baylor University, 101 Bagby Avenue, Waco, TX76706
}

(Dated: October 31, 2018)

\begin{abstract}
The gravitational collapse of a spherically symmetric star, made of a dust fluid, $\rho_{D M}$, in a background of dark energy, $p=w \rho,(w<-1 / 3)$, is studied. It is found that when only dark energy is present, black holes are never formed. When both of them are present, black holes can be formed, due to the condensation of the dust fluid. Initially the dust fluid may not play an important role, but, as time increases, it will dominate the collapse and finally leads to formation of black holes. This result remains true even when the interaction between the dust fluid and dark energy does not vanish. When $w<-1$ (phantoms), some models can also be interpreted as representing the death of a white hole that ejects both dust and phantoms. The ejected matter re-collapses to form a black hole.

PACS numbers: 97.60.-s, 95.35.+d, 97.60.Lf, 98.80.Cq
\end{abstract}

\section{INTRODUCTION}

Over the past decade, one of the most remarkable discoveries is that our universe is currently accelerating. This was first observed from high red shift supernova Ia [1] and confirmed later by cross checks from the cosmic microwave background radiation 2] and large scale structure [3].

In Einstein's general relativity, in order to have such an acceleration, one needs to introduce a component to the matter distribution of the universe with a large negative pressure. This component is usually referred to as dark energy. Astronomical observations indicate that our universe is flat and currently consists of approximately $2 / 3$ dark energy and $1 / 3$ dark matter. The nature of dark energy as well as dark matter is unknown, and many radically different models have been proposed, such as, a tiny positive cosmological constant, quintessence, phantoms, Chaplygin gas, and dark energy in brane worlds, among many others [See the review articles $[4,[5,66,7,6,[9]$, and references therein].

On the other hand, another very important issue in gravitational physics is black holes and their formation in our universe. Although it is generally believed that on scales much smaller than the horizon size the fluctuations of dark energy itself are unimportant [10], their effects on the evolution of matter overdensities may be significant 11]. Then, a natural question is how dark energy affects the process of the gravitational collapse of a star. It is known that dark energy exerts a repulsive force on its surrounding, and this repulsive force may prevent the star from collapse. Indeed, there are speculations that a massive star doesn't simply collapse to form a black hole, instead, to the formation of stars that contain dark energy. As a result, black holes may not exist at all 12]. Another related issue is that how dark energy affects already-formed black holes (if they indeed exist in our universe). Recently, it was shown that the mass of a black hole decreases due to phantom energy accretion and tends to zero when the Big Rip approaches [13].
In this paper, we shall study the formation of black holes from the gravitational collapse of a dust cloud in the background of dark energy, here "dust cloud" means a cloud made of matter with zero pressure. Thus, it includes the dark matter as a particular case. In particular, in Sec. II we consider the collapse of a homogeneous and isotropic star with finite radius, and develop the general formulas for the problem. The formation of black holes is identified by the development of apparent horizons. In Sec. III we consider the gravitational collapse of dark energy and dust cloud separately, in order to study the different roles that they may play during the collapse. We show explicitly that the collapse of the dark energy alone can never form black holes. In Sec. IV, we study the collapse of a dust cloud in the presence of dark energy, but there is no interaction between them except for the gravitational one. It is found that black hole can be formed due to the condensation of the dust cloud. In Sec. V, we study the collapse of a dust cloud and dark energy when the interaction between them does not vanish. We find that such interaction does not change the output significantly. In particular, black hole can still be formed. The paper is closed with Sec. VI, where our main conclusions are presented.

\section{FIELD EQUATIONS FOR COLLAPSING SPHERICAL STAR OF A DUST CLOUD}

In this section, we consider the gravitational collapse of a spherically symmetric star with finite thickness, which is made of a dust cloud in a background of dark energy. Let us first divide the spacetime into three different regions, $\Sigma$ and $V^{ \pm}$, where $\Sigma$ denotes the surface of the star, and $V^{-}\left(V^{+}\right)$the interior (exterior) of the star [cf. Fig. 1]. For the sake of simplicity, we assume that the spacetime inside the star is homogeneous and isotropic, similar to the Oppenheimer-Synyder (OS) model [14], historically the first model for gravitational collapse. Then, the 
spacetime inside the star is described by the metric

$$
d s_{-}^{2}=d t^{2}-a^{2}(t)\left(d r^{2}+r^{2} d \Omega^{2}\right)
$$

where $d \Omega^{2} \equiv d \theta^{2}+\sin ^{2} \theta d \varphi^{2}$, and $a(t)$ is an arbitrary function of $t$ only. Although this is a very ideal case, we do believe that this captures the main features of gravitational collapse in the background of dark energy, similar to the OS model that gives most of the main properties of a collapsing star in an otherwise flat background [15]. Since the matter fields are comoving in the spacetime described by metric (2.1), one may choose the surface $\Sigma$ to be described by

$$
\left.r\right|_{\Sigma}=\text { Constant, say, } r_{\Sigma}
$$

in the $x^{-\mu}$-coordinates. Introducing the intrinsic coordinates $\xi^{a}$ on $\Sigma$ by $\xi^{a} \equiv(\tau, \theta, \varphi)$, the metric on $\Sigma$ can be cast in the form,

$$
\left.d s^{2}\right|_{\Sigma} \equiv \gamma_{a b} d \xi^{a} d \xi^{b}=d \tau^{2}-R^{2}(\tau) d \Omega^{2},
$$

where

$$
\tau=t, \quad R(\tau)=r_{\Sigma} a(\tau)
$$

Then, the normal vector $n_{\alpha}^{-}$to the surface $\Sigma$ is given by,

$$
n_{\alpha}^{-}=\frac{1}{a(t)} \delta_{\alpha}^{r}
$$

and the non-zero components of the corresponding extrinsic curvature, defined by

$$
K_{a b}=-n_{\alpha}\left(\frac{\partial^{2} x^{\alpha}}{\partial \xi^{a} \partial \xi^{b}}+\Gamma_{\beta \delta}^{\alpha} \frac{\partial x^{\beta}}{\partial \xi^{a}} \frac{\partial x^{\delta}}{\partial \xi^{b}}\right),
$$

are given by,

$$
K_{\theta \theta}^{-}=\sin ^{-2} \theta K_{\phi \phi}^{-}=r_{\Sigma} a(t) .
$$

On the other hand, the metric outside the collapsing cloud in general can be cast in the form,

$$
d s_{+}^{2}=A^{2}(T, R) d T^{2}-B^{2}(T, R)\left(d R^{2}+R^{2} d \Omega^{2}\right),
$$

where $x^{+\mu} \equiv\{T, R, \theta, \phi\}$ denote the coordinates used outside of the collapsing dust cloud. The surface $\Sigma$ in the $x^{+\mu}$-coordinates can be expressed as

$$
R=R_{0}(T),
$$

for which the normal vector to $\Sigma$ is given by

$$
n_{\alpha}^{+}=\frac{A B}{\sqrt{A^{2}-R_{0}^{\prime 2} B^{2}}}\left\{\delta_{\alpha}^{R}-R_{0}^{\prime} \delta_{\alpha}^{T}\right\},
$$

where $R_{0}^{\prime} \equiv d R_{0} / d T$. Then, the junction conditions $\left.d s_{-}^{2}\right|_{\Sigma^{-}}=\left.d s_{+}^{2}\right|_{\Sigma^{+}}$require

$$
\begin{aligned}
\frac{d T}{d t} & =\frac{1}{\sqrt{A^{2}-R_{0}^{\prime} B^{2}}}, \\
r_{\Sigma} a(t) & =R_{0}(T) B\left(T, R_{0}(T)\right) .
\end{aligned}
$$

The non-vanishing components of the extrinsic curvature, $K_{a b}^{+}$, are given by,

$$
\begin{aligned}
K_{\tau \tau}^{+}= & \frac{A B}{\left(A^{2}-R_{0}^{\prime}{ }^{2} B^{2}\right)^{3 / 2}}\left\{\frac{B B_{, T}}{A^{2}} R_{0}^{\prime}{ }^{3}\right. \\
& +\left(2 \frac{A_{, R}}{A}-\frac{B, R}{B}\right) R_{0}^{\prime 2}+\left(\frac{A_{, T}}{A}-2 \frac{B, T}{B}\right) R_{0}^{\prime} \\
& \left.-R_{0}^{\prime \prime}-\frac{A A_{, R}}{B^{2}}\right\}, \\
K_{\theta \theta}^{+}= & \sin ^{-2} \theta K_{\phi \phi}^{-}=\frac{A B^{2} R_{0}}{\left(A^{2}-R_{0}^{\prime}{ }^{2}\right)^{1 / 2}} \\
& \times\left(\frac{R_{0} B_{, T}}{A^{2}} R_{0}^{\prime}+\frac{B+R_{0} B_{, R}}{B^{2}}\right) .
\end{aligned}
$$

Depending on the choice of the spacetime outside the star, there are two possibilities [16]: one is that the induced metrics $\gamma_{a b}^{ \pm}$are continuous across $\Sigma$, but not $K_{a b}^{ \pm}$. In this case, one can show that $\Sigma$ is an energy layer, and an infinitely thin matter shell appears on $\Sigma$. The other possibility is that both $\gamma_{a b}^{ \pm}$and $K_{a b}^{ \pm}$are continuous across $\Sigma$. In this case, Israel junction conditions tell us that no such a shell appears on $\Sigma$. Once the spacetime inside the surface is fixed, that whether a thin shell appears on $\Sigma$ or not is completely determined by the spacetime outside the star. In this paper, we shall consider only the latter case, that is,

$$
\begin{aligned}
K_{\tau \tau}^{+} & =K_{\tau \tau}^{-}, \\
K_{\theta \theta}^{+} & =K_{\theta \theta}^{-} .
\end{aligned}
$$

Eqs. 2.11), (2.12), 2.15) and (2.16) are the four equations that the four functions $T(t), \quad R_{0}(T(t))$, $A\left(T(t), R_{0}(T(t))\right)$ and $B\left(T(t), R_{0}(T(t))\right)$ must satisfy on the boundary $\Sigma$. Once the dependence of $A$ and $B$ on $T$ and $R$ are given, these equations will uniquely determine the time evolution of $T, R_{0}, A$ and $B$ along the hypersurface $\Sigma$.

However, such dependence may not be always consistent with these four equations. For example, it is wellknown that if the spacetime outside the collapsing cloud is empty, then there is no way to do the matching for the case where the dark energy does not vanish $(p \neq 0)$ [17]. An interesting case is that the spacetime outside the dust cloud is described by the McVittie solutions [18], which in general describes a Schwarzschild black hole in the background of the FRW cosmology. This is under our current investigations [19].

In this paper we shall focus our attention mainly in the spacetime inside the star. The main reasons for doing so are two-fold. First, such a matching, in general, is not unique, as shown above. In principle, there are infinite ways to do the matching. Second, if the collapsing star finally forms a black hole, an apparent horizon must develop inside the star, and there exists a moment at which the whole star collapses inside the apparent horizon. Clearly, this moment is exactly $\tau_{A H}$ [cf. Fig. 1], 
given below by Eq.(2.27). On the other hand, if the collapse doesn't form a black hole, apparent horizons will never be formed inside the star, and the star will not be trapped at any moment. As a result, the condition (2.29) remains true all the time. Therefore, the analysis whether a black hole is formed reduces to the one whether apparent horizons develop inside the star, that is, whether Eq. (2.27) has real solutions or not. It does not depend on the matching, neither on which spacetime outside the star is chosen, although the matching and spacetime outside the star do affect the total mass of the black hole and the global structure of the spacetime. This can be seen more clearly in the model to be presented in Sec. III. 1, which is essentially the OS model. As we mentioned above, in this paper we are mainly concerned with whether or not a black hole can be formed from the collapse of a dust cloud in the background of dark energy, therefore, in the following we shall consider only the spacetime inside the star.

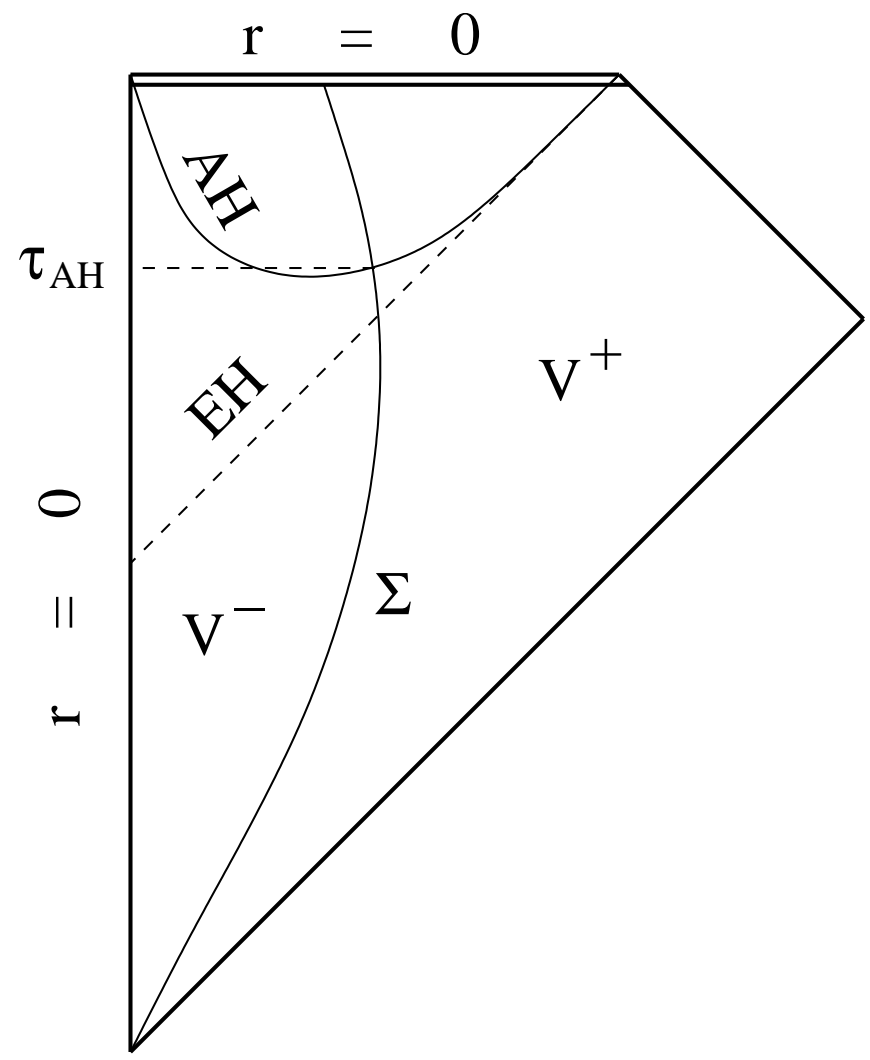

FIG. 1: The Penrose diagram of a collapsing star with a finite thickness that finally forms a black hole. The line $\Sigma$ represent the history of the surface of the star. The curved line $A H$ denotes the location of the apparent horizon, while the dashed line $E H$ the location of the event horizon, which is known only at the end of the collapse. The crossing point, $\tau=$ $\tau_{A H}$, denotes the moment when the whole star collapses inside the apparent horizon. Then, $M\left(\tau_{A H}\right)$, defined by Eq.(2.26), represents the total contribution of the internal part of the star to the total mass of the black hole.

The energy-momentum tensor (EMT) $T_{\mu \nu}^{-}$inside the star is given by

$$
T_{\mu \nu}^{-}=\left(\rho_{D M}+\rho+p\right) u_{\mu}^{-} u_{\nu}^{-}-p g_{\mu \nu}^{-},
$$

where $\rho_{D M}$ denotes the energy density of the dust cloud, and $\rho$ and $p$ are, respectively, the energy density and pressure of the dark energy, while $u_{\mu}^{-}$is their four-velocity. Since the fluid is comoving with the coordinates, we have $u_{\mu}^{-}=\delta_{\mu}^{t}$. Then, the Einstein field equations $G_{\mu \nu}^{-}=\kappa T_{\mu \nu}^{-}$ read,

$$
\begin{aligned}
& \frac{\ddot{a}}{a}=-\frac{1}{6} \kappa\left(\rho_{D M}+\rho+3 p\right), \\
& \frac{\dot{a}^{2}}{a^{2}}=\frac{1}{3} \kappa\left(\rho_{D M}+\rho\right),
\end{aligned}
$$

where $\dot{a} \equiv d a(t) / d t$. The interaction between the dust cloud and dark energy is given by the conservation law, $T_{\mu \nu ; \lambda}^{-} g^{-\nu \lambda}=0$, which in the present case reads

$$
\begin{aligned}
& \dot{\rho}_{D M}+3\left(\frac{\dot{a}}{a}\right) \rho_{D M}=Q, \\
& \dot{\rho}+3\left(\frac{\dot{a}}{a}\right)(\rho+p)=-Q,
\end{aligned}
$$

where $Q=Q(t)$ denotes the interaction between the dust cloud and dark energy. Since in this paper we are mainly concerned with gravitational collapse, we assume that

$$
\dot{a}<0 \text {. }
$$

The formation of a black hole is identified by the development of an apparent horizon $(\mathrm{AH})$, on which we have

$$
R_{, \alpha} R_{, \beta} g^{-\alpha \beta}=(r \dot{a})^{2}-1=0,
$$

where ()$_{,} \equiv \partial() / \partial x$, and

$$
R(t, r) \equiv r a(t),
$$

denotes the geometrical radius of the two-spheres, $t, r=$ Const.

Another important quantity to describe the collapse is the mass function $m(t, r)$, defined by

$$
m(t, r) \equiv \frac{1}{2} R\left(1+R_{, \alpha} R_{, \beta} g^{\alpha \beta}\right)=\frac{1}{2} r^{3} a \dot{a}^{2},
$$

which can be interpreted as the total mass inside the radius $r$ at the moment $t$. This definition was first introduced by Cahill and McVittie [20], and has been widely used since then [21]. In asymptotically flat spacetimes, it gives the correct Bondi mass at infinity [22]. On the surface $r=r_{\Sigma}$, Eq.(2.25) gives the total mass of the collapsing star at the moment $\tau$ [23],

$$
M(\tau) \equiv m\left(r_{\Sigma}, \tau\right)=\frac{1}{2} R(\tau) \dot{R}^{2}(\tau) .
$$

Assuming that the time when the whole star collapses inside the apparent horizon is $\tau_{A H}$ [cf. Fig. 1], from Eq. (2.23) we have

$$
\left.\dot{R}^{2}\left(\tau_{A H}\right)\right|_{\Sigma}=1
$$


Then, the total contribution of the collapsing star to the mass of the black hole is given by

$$
M_{B H}=M\left(\tau_{A H}\right) \text {. }
$$

If no matter continuously falls into the black hole from outside of the star after the moment $\tau_{A H}$, we can see that Eq.(2.28) gives the total mass of the black hole.

Since in this paper we are mainly interested in the formation of black holes due to the gravitational collapse of the star, we assume that at the initial of the collapse, $\tau=\tau_{i}$, the star is not trapped, that is,

$$
\left.R_{, \alpha} R_{, \beta} g^{\alpha \beta}\right|_{\tau=\tau_{i}}=\left(r_{\Sigma} \dot{a}\left(\tau_{i}\right)\right)^{2}-1<0 .
$$

Once we have the general formulas, in the following three sections, $I I I-V$, we shall consider some specific models. Although most of the solutions are already known in the context of cosmology, the studies of them in the context of gravitational collapse are new, and, as we shall see below, shall lead to important conclusions regarding to whether black holes can be formed in the background of dark energy.

\section{GRAVITATIONAL COLLAPSE OF A DUST CLOUD OR DARK ENERGY}

In this section, we consider a collapsing dust cloud and dark energy separately, in order to see the different roles that they may play during the collapse.

\section{A. Gravitational Collapse of a dust cloud}

In this case we assume that

$$
\rho_{D M} \neq 0, \quad \rho=0=p .
$$

That is, the collapsing star consists of only a dust cloud. Historically, this was the first example to study gravitational collapse, which leads to the formation of black holes [14]. In the following, we shall briefly review the main properties of the collapse in the framework given above, so we can see clearly the role that the dust cloud plays during the collapse. From Eq. 2.20) we find that $\rho_{D M}=\rho_{D M}^{0} / a^{3}$, where $\rho_{D M}^{0}$ is an integration constant. Then, Eqs.(2.19) and (2.22) yield

$$
a(t)=a_{0}\left(t_{0}-t\right)^{2 / 3},
$$

where $a_{0} \equiv\left(3 \kappa \rho_{D M}^{0} / 4\right)^{1 / 3}$, and $t_{0}$ is another integration constant. Hence, the physically relevant quantities in this case are given by

$$
\begin{aligned}
\rho_{D M} & =\frac{4}{3 \kappa\left(t_{0}-t\right)^{2}} \\
\dot{R}(\tau) & =-\frac{2}{3} R_{0}\left(\tau_{0}-\tau\right)^{-1 / 3} \\
M(\tau) & =\frac{2}{9} R_{0}^{3}
\end{aligned}
$$

where $R_{0} \equiv r_{\Sigma} a_{0}$ and $\tau_{0}=t_{0}$. Assuming that the star starts to collapse at the moment $\tau=\tau_{i}$, where the condition 2.29) holds, that is, the star is not initially trapped, then from Fig. 2 and the expressions of Eq.(3.3) we can see that the star shall collapse until the moment $\tau=\tau_{0}$, where a spacetime singularity is finally formed. This singularity is not naked, because before it is formed, an apparent horizon is already formed at $\tau_{A H}$, where

$$
\tau_{A H}=\tau_{0}-\left(\frac{2 R_{0}}{3}\right)^{3}<\tau_{0}
$$

Thus, in this case the collapse actually forms black holes. It is interesting to note that the total mass of the star in the present case remains constant during the whole process of the collapse. This is understandable. As shown in [14], in the present case the star can be smoothly matched to the Schwarzschild vacuum solution without a thin shell appearing on the surface of the collapsing star. Then, we have $M_{B H}=2 R_{0}^{3} / 9$, which is the mass of the Schwarzschild black hole.

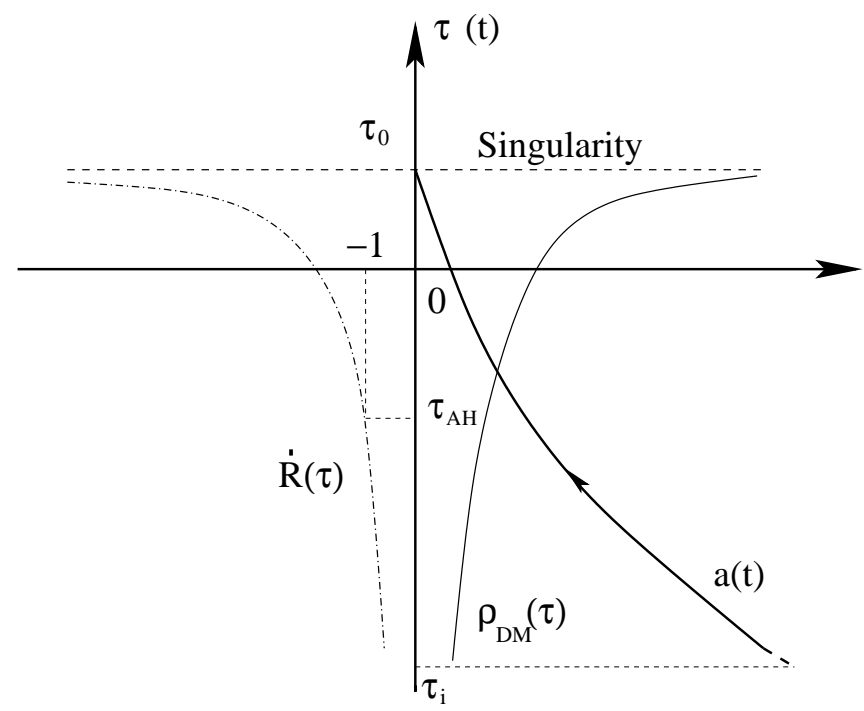

FIG. 2: The star with radius $r_{\Sigma}$, made of a dust cloud, starts to collapse at the moment $\tau=\tau_{i}$ in an otherwise flat spacetime. At the moment $\tau=\tau_{A H}$ an apparent horizon develops, and whereby a black hole is formed. From that moment on the entire star is inside the black hole.

From this example, we can also see clearly that to study whether a collapsing star forms a black hole or not now indeed becomes to study whether an apparent horizon develops in the internal region of the star.

\section{B. Gravitational Collapse of Dark Energy}

To study the effects of dark energy on gravitational collapse, we first consider the case where

$$
\rho_{D M}=0, \quad p=w \rho \neq 0
$$


where $w$ is a non-zero constant. When $w<-1 / 3$ the strong energy condition is not satisfied [24], and the fluid is said to be made of dark energy. It can be shown that the solution in this case is given by

$$
\begin{aligned}
a(t) & =a_{0}\left(t_{0}-t\right)^{\frac{2}{3(1+w)}} \\
\rho(t) & =\frac{4}{3 \kappa(1+w)^{2}\left(t_{0}-t\right)^{2}}, \\
\dot{R}(\tau) & =-\frac{2 R_{0}}{3(1+w)}\left(\tau_{0}-\tau\right)^{-\frac{1+3 w}{3(1+w)}}
\end{aligned}
$$

for $w>-1$,

$$
\begin{aligned}
a(t)= & a_{0} \exp \left\{\left(\frac{\rho_{0} \kappa}{3}\right)^{1 / 2}\left(t_{0}-t\right)\right\}, \\
\rho(t)= & \rho_{0}, \\
\dot{R}(\tau)= & -R_{0}\left(\frac{\rho_{0} \kappa}{3}\right)^{1 / 2} \\
& \times \exp \left\{\left(\frac{\rho_{0} \kappa}{3}\right)^{1 / 2}\left(t_{0}-t\right)\right\},
\end{aligned}
$$

for $w=-1$, and

$$
\begin{aligned}
a(t) & =a_{0}\left(t-t_{0}\right)^{\frac{2}{3(1+w)}} \\
\rho(t) & =\frac{4}{3 \kappa(1+w)^{2}\left(t-t_{0}\right)^{2}} \\
\dot{R}(\tau) & =-\frac{2 R_{0}}{3(|w|-1)}\left(\tau-\tau_{0}\right)^{-\frac{3|w|-1}{3(|w|-1)}}
\end{aligned}
$$

for $w<-1$.

When $w>-1 / 3$, for which all the energy conditions are satisfied, the collapse is quite similar to that of the dust cloud, studied in the last subsection. In particular, a black hole is always formed, and the formation of an apparent horizon happens at,

$$
\tau_{A H}=\tau_{0}-\left(\frac{2 R_{0}}{3(1+w)}\right)^{\frac{3(1+w)}{1+3 w}}<\tau_{0},
$$

where $\tau=\tau_{0}>\tau_{A H}$ is the moment when the spacetime singularity develops. The total mass of the star is given by

$$
M(\tau)=\frac{2 R_{0}^{3}}{9(1+w)^{2}}\left(\tau_{0}-\tau\right)^{-\frac{2 w}{1+w}},
$$

from which we can see that $M(\tau) \rightarrow \infty$ for $w>0$ and $M(\tau) \rightarrow 0$ for $-1 / 3<w<0$, as the spacetime singularity at $\tau=\tau_{0}$ approaches.

It should be noted that, although $M(\tau) \rightarrow 0$ for $-1 / 3<w<0$, the energy density $\rho(t) \rightarrow \infty$, as $\tau \rightarrow \tau_{0}$, as one can see from Eq.(3.6). Then, the spacetime is still singular at $\tau_{0}$ for $w \in(-1 / 3,0)$.

When $w=-1 / 3$, from Eq.(3.6) we find $\dot{R}(\tau)=-R_{0}$. Thus, if the collapsing star initially is not trapped, it will remain so until a spacetime singularity develops at the moment $t=t_{0}$, which is spacelike. It should be noted that in this case all the energy conditions are satisfied, and the total mass of the star is given by

$$
M(\tau)=\frac{1}{2} R_{0}^{3}\left(\tau_{0}-\tau\right)
$$

which vanishes as $\tau \rightarrow \tau_{0}$, although $\rho \simeq\left(t_{0}-t\right)^{-2} \rightarrow \infty$ in this limit.

When $-1<w<-1 / 3$, from Eq.(3.6) we find that

$$
\begin{aligned}
\dot{R}(\tau) & =-\frac{2 R_{0}}{3(1-|w|)}\left(\tau_{0}-\tau\right)^{\frac{3|w|-1}{3(1-|w|)}} \\
M(\tau) & =\frac{2 R_{0}^{3}}{9(1-|w|)^{2}}\left(\tau_{0}-\tau\right)^{\frac{2|w|}{1-|w|}} \\
\rho(t) & =\frac{4}{3 \kappa(1+w)^{2}\left(t_{0}-t\right)^{2}} .
\end{aligned}
$$

The evolution of these quantities with time $\tau$ are shown in Fig. 3. From there we can see that if the star is not trapped initially, it will never become trapped in the future. If the star is trapped initially, it will become untrapped at the moment

$$
\tau_{N}=\tau_{0}-\left(\frac{3(1-|w|)}{2 R_{0}}\right)^{\frac{3(1-|w|)}{3|w|-1}} .
$$

The collapse always forms a spacelike singularity with zero mass at the moment $\tau=\tau_{0}$.

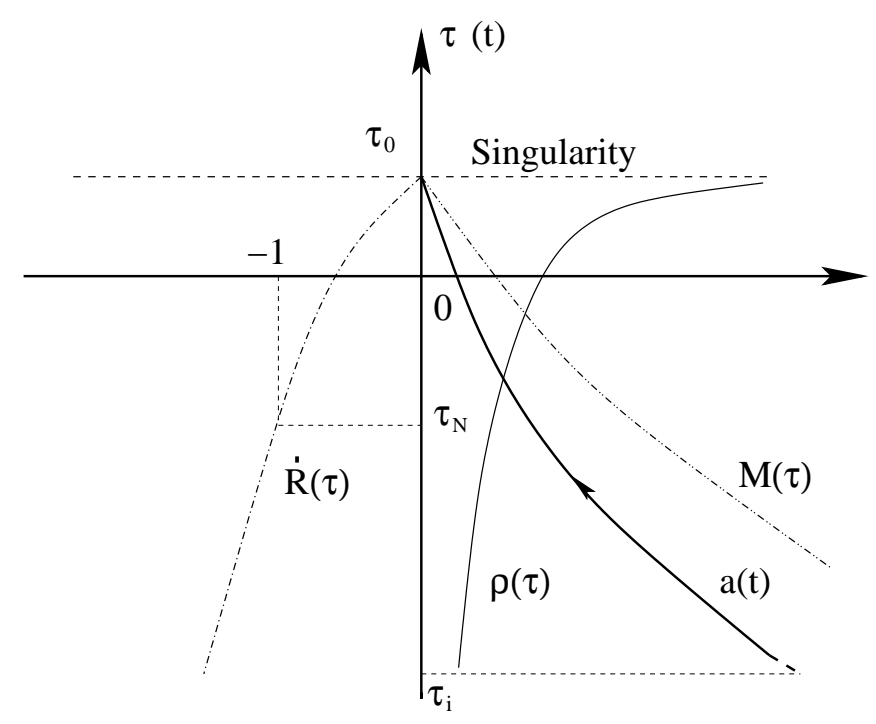

FIG. 3: The star with radius $r_{\Sigma}$, made of dark energy with $-1<w<-1 / 3$, starts to collapse at the moment $\tau=\tau_{i}$. If the star is trapped initially, it will become untrapped at $\tau=\tau_{N}$. If it is not trapped initially, it will remain so in the future. The collapse always develops a spacelike singularity at $\tau=\tau_{0}$ with zero mass.

When $w=-1$, the solution given by Eq. (3.7) represents the de Sitter space, and the properties of this spacetime is well-known [24], so in the following we don't consider it any more. 


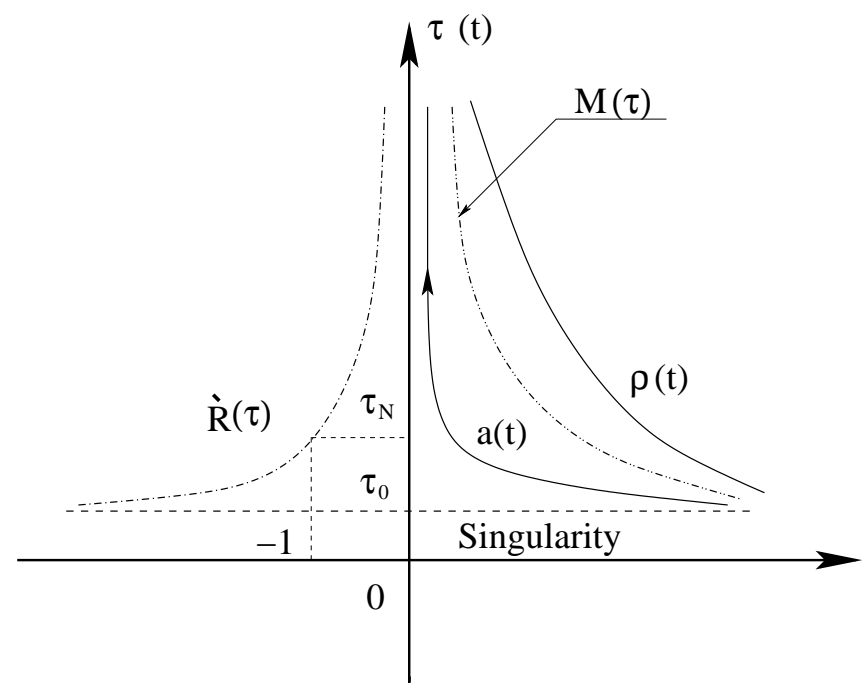

FIG. 4: The star with radius $r_{\Sigma}$, made of dark energy with $w<-1$, starts to collapse at the moment $\tau=\tau_{i}$. The total mass of the collapsing star will be eventually zero, so that the spacetime is finally flat.

When $w<-1$, from Eq. (3.8) we find that

$$
M(\tau)=\frac{2 R_{0}{ }^{3}}{9(|w|-1)^{2}}\left(\tau-\tau_{0}\right)^{-\frac{2|w|}{|w|-1}}= \begin{cases}0, & \tau \rightarrow \infty \\ \infty, & \tau \rightarrow \tau_{0} .\end{cases}
$$

Some relevant quantities are plot in Fig. 4, from which we can see that if $R_{, \alpha} R^{, \alpha}=\dot{R}^{2}-1<0$ initially, it will remain so all the time. That is, in this case the collapse never forms a black hole, neither does a spacetime singularity. If it collapses initially with $R_{, \alpha} R^{, \alpha}=\dot{R}^{2}-1>0$, the star will become untrapped at the moment $\tau=\tau_{N}$, where

$$
\tau_{N}=\tau_{0}+\left(\frac{2 R_{0}}{3(|w|-1)}\right)^{\frac{3(|w|-1)}{3|w|-1}} .
$$

Thus, the total mass and energy density $\rho(t)$ of the collapsing star decrease as time increases, and finally become zero in the limit $\tau \rightarrow \infty$. Although $a(t=\infty)=0$, no spacetime singularity is formed there, as one can see from Eq. (3.8).

In review of all the above, we can see that due to its large negative pressure, the dark energy alone never collapses to form black holes.

\section{GRAVITATIONAL COLLAPSE OF A DUST CLOUD AND DARK ENERGY: WITHOUT INTERACTION $Q=0$}

When $Q=0$, Eqs.(2.20) and (2.21) have the solutions

$$
\begin{aligned}
\rho_{D M} & =\frac{\rho_{D M}^{0}}{a^{3}}, \\
\rho & =\frac{\rho_{0}}{a^{3(1+w)}},
\end{aligned}
$$

where $\rho_{D M}^{0}$ and $\rho_{0}$ are positive constants. Clearly, when $w<-1$ the spacetime will be singular at both $a=0$ and $a=\infty$. From Eqs.(2.19) and (2.22) we obtain

$$
\frac{d y}{\sqrt{1+y^{-2 w}}}=-\beta d t
$$

where

$y \equiv\left(\frac{\rho_{0}}{\rho_{D M}^{0}}\right)^{-\frac{1}{2 w}} a^{3 / 2}, \quad \beta \equiv\left(\frac{\rho_{0}}{\rho_{D M}^{0}}\right)^{-\frac{1}{2 w}}\left(\frac{3}{4} \kappa \rho_{D M}^{0}\right)^{1 / 2}$.

$$
\text { A. } w=-\frac{1}{2}
$$

When $w=-1 / 2$, Eq. (4.2) has the solution

$$
\begin{aligned}
a(t) & =a_{0}\left(\left(t_{0}-t\right)^{2}-A^{2}\right)^{2 / 3}, \\
a_{0} & \equiv\left(\frac{3 \kappa \rho_{0}}{16}\right)^{2 / 3}, \quad A \equiv\left(\frac{16 \rho_{D M}^{0}}{3 \kappa \rho_{0}^{2}}\right)^{1 / 2} .
\end{aligned}
$$

Then, we obtain

$$
\begin{aligned}
\rho_{D M} & =\frac{\rho_{D M}^{0}}{\left(\frac{3 \kappa \rho_{0}}{16}\right)^{2}\left[\left(t_{0}-t\right)^{2}-A^{2}\right]^{2}}, \\
\rho & =\frac{16}{3 \kappa\left[\left(t_{0}-t\right)^{2}-A^{2}\right]}, \\
\dot{R}(\tau) & =-\frac{4}{3} R_{0} \frac{\left(\tau_{0}-\tau\right)}{\left[\left(\tau_{0}-\tau\right)^{2}-A^{2}\right]^{1 / 3}}, \\
M(\tau) & =\frac{8}{9} R_{0}^{3}\left(\tau_{0}-\tau\right)^{2} .
\end{aligned}
$$

From the above expressions we can see that the spacetime is singular at $t_{s}$, where $t_{s}=t_{0}-A$. We also have

$$
\begin{aligned}
\dot{R}(\tau) & =-\frac{4}{3} R_{0} \frac{\left(\tau_{0}-\tau\right)}{\left[\left(\tau_{0}-\tau\right)^{2}-A^{2}\right]^{1 / 3}} \\
& =\left\{\begin{array}{l}
-\infty, \quad \tau \rightarrow-\infty, \tau_{s}, \\
-B, \quad \tau=\tau_{\text {min. }},
\end{array}\right.
\end{aligned}
$$

where

$$
\tau_{\text {min. }} \equiv \tau_{0}-\sqrt{3} A, \quad B \equiv \frac{4^{2 / 3}}{3^{1 / 3}} R_{0} A^{1 / 3},
$$

as shown by Fig. 5. Thus, if $B>1$ we have $R_{, \alpha} R^{, \alpha}>0$ all the time, and the star is trapped during the whole process of collapse. In order to have $R_{, \alpha} R^{, \alpha}<0$ initially, we must choose $r_{\Sigma}, \rho_{D M}^{0}$ and $\rho_{0}$ such that

$$
B<1 \text {. }
$$

Once this condition is satisfied, from Fig. 5 we can see that as long as $\tau_{i}>\tau_{A H}^{-}$, the collapsing star will not be trapped at the initial. However, as the time increases, the dust cloud becomes dominant over the dark energy, 
and an apparent horizon will finally develop at the moment $\tau=\tau_{A H}^{+}$, where $\tau_{A H}^{ \pm}$are the two real roots of the equation,

$$
\left(\tau_{0}-\tau\right)^{3}-\left(\frac{3}{4 R_{0}}\right)^{3}\left(\tau_{0}-\tau\right)^{2}+A^{2}\left(\frac{3}{4 R_{0}}\right)^{3}=0
$$

with $\tau_{A H}^{+}>\tau_{A H}^{-}$. This can be seen clearly from,

$$
\frac{\rho_{D M}}{\rho}=\frac{16 \rho_{D M}^{0}}{3 \kappa \rho_{0}^{2}\left[\left(t_{0}-t\right)^{2}-A^{2}\right]}= \begin{cases}0, & t \rightarrow-\infty \\ \infty, & t \rightarrow t_{s} .\end{cases}
$$

Thus, a spacetime singularity develops at $t_{s}$. From Eq.4.5 we can see that the mass of a such formed black hole is finite.

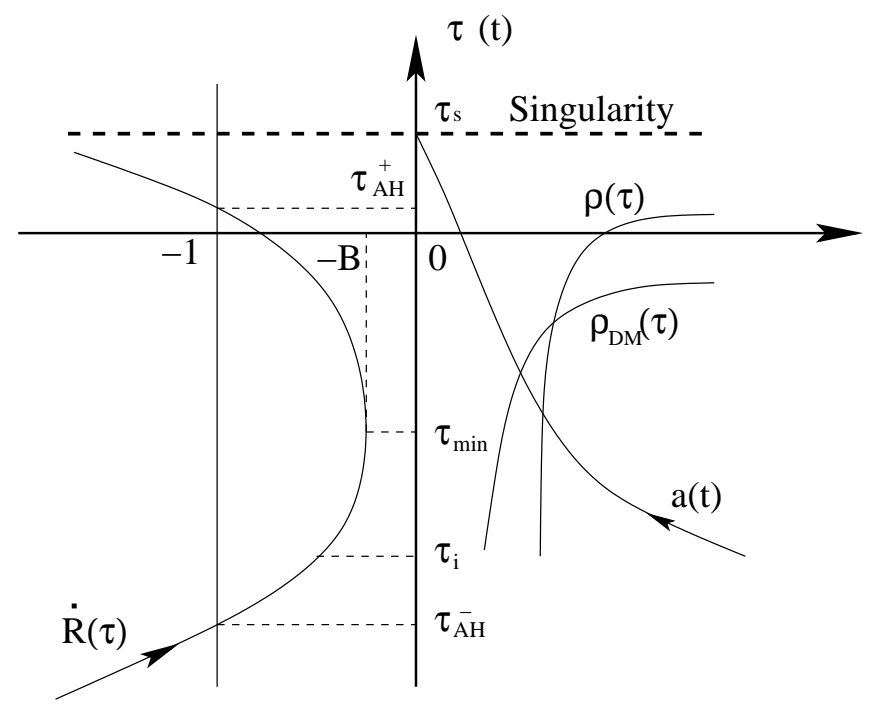

FIG. 5: The collapsing star with radius $r_{\Sigma}$, made of a dust cloud in the background of dark energy without interaction, $Q=0$, for $B<1$. It starts to collapse at the moment $\tau=\tau_{i}$. As time increases, the dust cloud becomes dominant, and an apparent horizon finally develops at $\tau_{A H}^{+}$, whereby a black hole is formed. From this moment on, the star collapses entirely inside the black hole, and at the moment $\tau_{s}$ a spacetime singularity develops.

\section{B. $w=-1$}

In this case, it can be shown that the solutions are given by

$$
\begin{aligned}
a(t) & =a_{0} \sinh ^{2 / 3}\left[\beta\left(t_{0}-t\right)\right], \\
\rho_{D M} & =\frac{\rho_{0}}{\sinh ^{2}\left[\beta\left(t_{0}-t\right)\right]}, \\
\rho & =\rho_{0},
\end{aligned}
$$

where $a_{0}$ is a positive constant, and

$$
\beta \equiv\left(\frac{3}{4} \kappa \rho_{0}\right)^{1 / 2}
$$

Then, we obtain

$$
\begin{aligned}
\dot{R}(\tau) & =-\frac{2}{3} \beta R_{0} \frac{\cosh \left[\beta\left(\tau_{0}-\tau\right)\right]}{\sinh ^{1 / 3}\left[\beta\left(\tau_{0}-\tau\right)\right]} \\
& = \begin{cases}-\infty, & \tau \rightarrow-\infty, \\
-\frac{4^{1 / 3}}{3^{1 / 2}} \beta R_{0}, & \tau=\tau_{\text {min. }}, \\
-\infty, & \tau=\tau_{0},\end{cases}
\end{aligned}
$$

where

$$
\tau_{\text {min. }} \equiv \tau_{0}-\frac{1}{\beta} \sinh ^{-1}\left(\frac{1}{\sqrt{2}}\right)
$$

The curve of $\dot{R}(\tau)$ versus $\tau$ is quite similar to that given in Fig. 5, except that now the spacetime singularity occurs at $\tau=\tau_{0}$. Thus, in order to have the collapsing star untrapped at the initial, we must choose the free parameters $a_{0}, r_{\Sigma}$ and $\rho_{0}$ such that

$$
\beta R_{0}<\frac{3^{1 / 2}}{4^{1 / 3}}
$$

Then, as shown by Fig. 5, choosing $\tau_{i}>\tau_{A H}^{-}$we can see that the solution can be interpreted as representing gravitational collapse of a dust cloud in the background of dark energy (in the present case it is the cosmological constant.). At initial the collapsing star is untrapped. However, as time increases, the dust cloud becomes dominant, and finally an apparent horizon develops at the moment $\tau=\tau_{A H}^{+}$, whereby a black hole is formed, where $\tau_{A H}^{ \pm}$now are given by

$$
\tau_{A H}^{ \pm} \equiv \tau_{0}-\frac{1}{\beta} \sinh ^{-1}\left(X^{ \pm 3 / 2}\right)
$$

and $X^{ \pm}$are the two real roots of the equation,

$$
X^{3}-\left(\frac{3}{2 \beta R_{0}}\right)^{2} X+1=0 .
$$

Note that the black hole formed in this case also has a finite non-zero mass, as we can see from the following expression,

$$
M(\tau)=\frac{2}{9} \beta R_{0}{ }^{3} \cosh ^{2}\left[\beta\left(\tau_{0}-\tau\right)\right] .
$$

$$
\text { C. } w<-1
$$

In general, the integration of Eq.(4.2) gives

$$
\text { y } F\left(\frac{1}{2},-\frac{1}{2 w} ; 1-\frac{1}{2 w} ;-y^{-2 w}\right)=-\beta\left(t-t_{0}\right),
$$

where $F(a, b ; c ; z)$ denotes the ordinary hypergeometric function with $F(a, b ; c ; 0)=1$. Thus, we find

$$
y \simeq-\beta\left(t-t_{0}\right) \sim 0
$$


as $t \rightarrow t_{0}$. On the other hand, using the relation [25],

$$
\begin{aligned}
F(a, b ; c ; z)= & \frac{\Gamma(c) \Gamma(b-a)}{\Gamma(b) \Gamma(c-a)}(-z)^{-a} \\
& \times F\left(a, 1-c+a ; 1-b+a ; \frac{1}{z}\right) \\
& +\frac{\Gamma(c) \Gamma(a-b)}{\Gamma(a) \Gamma(c-b)}(-z)^{-b} \\
& \times F\left(b, 1-c+b ; 1-a+b ; \frac{1}{z}\right),
\end{aligned}
$$

we find

$$
\begin{aligned}
y \quad & F\left(\frac{1}{2},-\frac{1}{2 w} ; 1-\frac{1}{2 w} ;-y^{-2 w}\right) \\
& \rightarrow \pi^{-1 / 2} \Gamma\left(1-\frac{1}{2 w}\right) \Gamma\left(\frac{1+w}{2 w}\right),
\end{aligned}
$$

as $y \rightarrow \infty$ for $w<-1$. Hence, we have

$$
y \rightarrow \infty,
$$

as $t \rightarrow t_{s}$, where

$$
t_{s} \equiv t_{0}-\frac{1}{\beta \pi^{1 / 2}} \Gamma\left(1-\frac{1}{2 w}\right) \Gamma\left(\frac{1+w}{2 w}\right) .
$$

Then, it can be seen that the curve of $a(t)$ versus $t$ is that given by Fig. 6. On the other hand, from Eq. (4.2) we also have

$$
\begin{aligned}
\dot{R}(\tau)= & -R_{0} \frac{\left[\rho_{D M}^{0}+\rho_{0} a^{-3 w}(\tau)\right]^{1 / 2}}{a^{1 / 2}(\tau)}, \\
\ddot{R}(\tau)= & -\left(\frac{1}{12} \kappa\right)^{1 / 2} \frac{R_{0}}{a^{2}} \\
& \times\left\{\rho_{D M}^{0}-(3|w|-1) \rho_{0} a^{-3 w}(\tau)\right\}, \\
M(\tau)= & \frac{1}{2} r_{\Sigma} R_{0}^{2}\left[\rho_{D M}^{0}+\rho_{0} a^{-3 w}(\tau)\right],
\end{aligned}
$$

where $R_{0} \equiv(\kappa / 3)^{1 / 2} r_{\Sigma}$. Thus, we find

$$
\dot{R}(\tau)= \begin{cases}-\infty, & \tau=\tau_{0} \\ -B, & \tau=\tau_{\min } \\ -\infty, & \tau=\tau_{s}\end{cases}
$$

where

$$
B \equiv R_{0} \frac{\left(\rho_{D M}^{0}+\frac{\rho_{D M}^{0}}{3|w|-1}\right)^{1 / 2}}{\left(\frac{\rho_{D M}^{0}}{(3|w|-1) \rho_{0}}\right)^{\frac{1}{6|w|}}}
$$

Clearly, for choice where $B<1$, there exists initial moment $\tau_{i}$ for which the collapsing star is not trapped at $\tau_{i}$. In fact, as long as $\tau_{A H}^{+}>\tau_{i}>\tau_{A H}^{-}$, the star is not trapped initially, as shown by Fig. 6 , where $\tau_{A H}^{ \pm}$are the two real roots of the equation $\dot{R}^{2}-1=0$. But, the collapse will eventually develop an apparent horizon at $\tau_{A H}^{+}$, whereby a black hole is formed. The spacetime becomes singularity at $\tau=\tau_{0}$ where $a\left(\tau_{0}\right)=0$, as shown by Eq.(4.20). From Eq.(4.25) we can see that such formed black holes have finite non-zero mass.

It is interesting to note that the solutions can also be interpreted as representing a white hole converting itself into a black hole 26], if we choose $\tau_{i}=\tau_{s}$, that is, the white hole evaporates through ejecting material, which will later re-collapse to form a black hole.

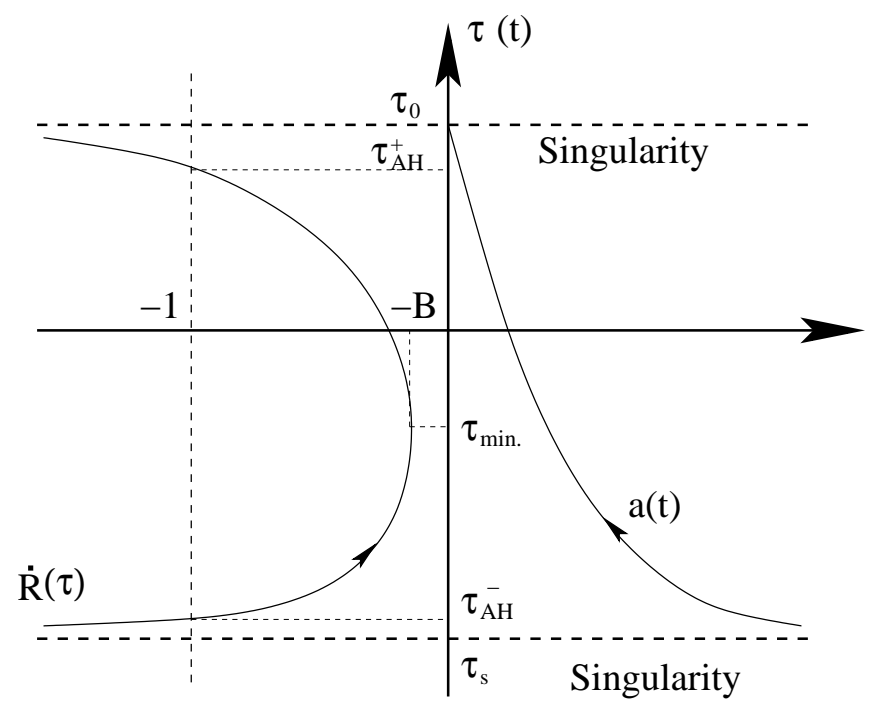

FIG. 6: The Curves of $\dot{R}(\tau)$ and $a(t)$ for $Q=0$ and $w<-1$.

\section{GRAVITATIONAL COLLAPSE OF A DUST CLOUD AND DARK ENERGY: WITH INTERACTION $Q \neq 0$}

Recently, we studied the interaction of a dust cloud and dark energy in the context of cosmology by assuming that [27]

$$
\frac{\rho}{\rho_{D M}}=A a^{3 n},
$$

where $A$ and $n$ are two arbitrary constants, subject to $A>0$. Assuming that the dark energy satisfies the equation of state $p=w \rho$ with $w$ being a constant, from Eqs. (2.20), (2.21) and (5.1) we obtain

$$
\begin{aligned}
\rho & =\frac{A \rho_{t}^{0} a^{3 n}}{a^{3}\left(1+A a^{3 n}\right)^{(w+n) / n}}, \\
\rho_{D M} & =\frac{\rho_{t}^{0}}{a^{3}\left(1+A a^{3 n}\right)^{(w+n) / n}},
\end{aligned}
$$

where $\rho_{t}^{0}$ is another positive constant. Substituting these into Eq.(2.19) and considering Eq.(2.22), we find

$$
\left(1+y^{2 n}\right)^{\frac{w}{2 n}} d y=-\beta d t
$$


where

$$
y \equiv A^{\frac{1}{2 n}} a^{3 / 2}, \quad \beta \equiv A^{\frac{1}{2 n}}\left(\frac{3}{4} \kappa \rho_{t}^{0}\right)^{1 / 2} .
$$

Hence, from Eq.(2.20) we have

$$
Q=-3 A(w+n)\left(\frac{\dot{a}}{a}\right) \frac{a^{3 n} \rho_{t}}{\left(1+A a^{3 n}\right)^{2}} .
$$

From Eq.(5.2), on the other hand, we obtain

$$
\begin{aligned}
\rho & = \begin{cases}a^{-3(1+w)}, & a \rightarrow \infty, \\
a^{3(n-1)}, & a \rightarrow 0,\end{cases} \\
\rho_{D M} & = \begin{cases}a^{-3(1+w+n)}, & a \rightarrow \infty, \\
a^{-3}, & a \rightarrow 0 .\end{cases}
\end{aligned}
$$

Therefore, the spacetime is always singular at $a=0$. When $w<-1$, it is also singular as $a \rightarrow \infty$.

\section{A. $n=1 / 2$}

When $n=1 / 2$, Eqs.(5.3) and (5.4) yield

$$
a(t)=a_{0}\left\{\left[\beta(1+w)\left(t_{0}-t\right)\right]^{\frac{1}{1+w}}-1\right\}^{2 / 3}, \quad(w \neq-1),
$$

for $w \neq-1$, and

$$
a(t)=a_{0}\left(e^{\beta\left(t_{0}-t\right)}-1\right)^{2 / 3}, \quad(w=-1),
$$

for $w=-1$, where $a_{0} \equiv A^{-2 / 3}$.

When $w>-1$, we find that

$$
a(t)= \begin{cases}\infty, & t \rightarrow-\infty \\ 0, & t=t_{s}\end{cases}
$$

with

$$
t_{s} \equiv t_{0}-\frac{1}{\beta(1+w)} .
$$

Then, from Eq.(5.2) we can see that the spacetime is singular at $t=t_{s}$. The nature of the singularity can be seen from $\dot{R}(\tau)$, given by

$$
\begin{aligned}
\dot{R}(\tau) & =-\frac{2}{3} \beta R_{0} \frac{\left[\beta(1+w)\left(\tau_{0}-\tau\right)\right]^{-\frac{w}{1+w}}}{\left\{\left[\beta(1+w)\left(\tau_{0}-\tau\right)\right]^{\frac{1}{1+w}}-1\right\}^{1 / 3}} \\
& = \begin{cases}0, & w>-1 / 3 \\
-2 \beta R_{0} / 3, & w=-1 / 3 \\
-\infty, & w<-1 / 3,\end{cases}
\end{aligned}
$$

as $\tau \rightarrow-\infty$. On the other hand, as $\tau \rightarrow \tau_{s}$ we have $\dot{R} \rightarrow-\infty$ for any value of $w$ with $w>-1$. Thus, the curve of $\dot{R}$ versus $\tau$ is that of Fig. 7 .

When $w>-1 / 3$, as shown by Fig. 7 , we can always choose an initial moment where $\dot{R}\left(\tau_{i}\right)>-1$ so

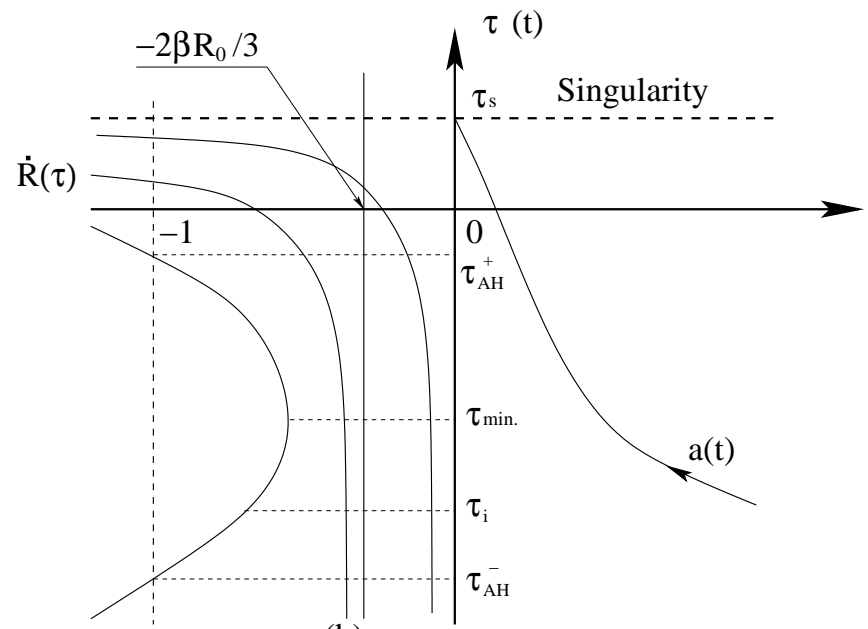

(c)

(b) (a)

FIG. 7: The Curve of $\dot{R}(\tau)$ versus $\tau$ for $Q \neq 0$. (a) $w>$ $-1 / 3$; (b) $w=-1 / 3$; and (c) $-1<w<-1 / 3$.

that the collapsing star is not trapped initially. As the star collapses, an apparent horizon develops at $\tau=\tau_{A H}^{+}$, whereby a black hole is formed. From this moment on, the collapsing star falls entirely inside the black hole. The total mass of it is finite,

$$
M(\tau)=\frac{2}{9} \beta^{2} R_{0}^{3}\left[\beta(1+w)\left(\tau_{0}-\tau\right)\right]^{-\frac{2 w}{1+w}} .
$$

When $w=-1 / 3$, the main properties of the solution are similar to these with $w>-1 / 3$, provided that $2 \beta R_{0} / 3<1$. When, $2 \beta R_{0} / 3>1$ the star will be trapped all time.

When $-1<w<-1 / 3$ we find that

$$
\dot{R}\left(\tau_{\text {min }} .\right)=-\frac{2}{3} \beta R_{0} \frac{(3|w|)^{|w|}}{(3|w|-1)^{(3|w|-1) / 3}},
$$

with

$$
\tau_{\text {min. }}=\tau_{0}-\frac{1}{\beta(1-|w|)}\left(\frac{3|w|}{3|w|-1}\right)^{1-|w|} .
$$

Thus, to have the collapsing star not trapped initially, we must require $\dot{R}\left(\tau_{\min }\right.$. $)>-1$. Then, for any given moment $\tau_{i}$, where $\tau_{A H}^{+}>\tau_{i}>\tau_{A H}^{-}$, the collapsing star is not trapped initially, but, as the star is collapsing, an apparent horizon will develop at $\tau_{A H}^{+}$, where $\tau_{A H}^{ \pm}$are the two real roots of the equation $\dot{R}(\tau)=-1$.

When $w=-1$, the solution is given by Eq.(5.8), from which we find that

$$
a(t)= \begin{cases}\infty, & t \rightarrow-\infty \\ 0, & t=t_{0} .\end{cases}
$$

From Eq.(5.2) we obtain

$$
\begin{aligned}
\rho & =A \rho_{t}^{0} \frac{1+A a^{3 / 2}}{a^{3 / 2}}, \\
\rho_{D M} & =\rho_{t}^{0} \frac{1+A a^{3 / 2}}{a^{3}} .
\end{aligned}
$$


Thus, the spacetime is singular at $t_{0}$, where $a\left(t_{0}\right)=0$. On the other hand, we also have

$$
\begin{aligned}
\dot{R}(\tau) & =-\frac{2}{3} \beta R_{0} \frac{e^{\beta\left(\tau_{0}-\tau\right)}}{\left[e^{\beta\left(\tau_{0}-\tau\right)}-1\right]^{1 / 3}} \\
\ddot{R}(\tau) & =-\frac{2}{9} \beta^{2} R_{0} \frac{e^{\beta\left(\tau_{0}-\tau\right)}}{\left[e^{\beta\left(\tau_{0}-\tau\right)}-1\right]^{4 / 3}}\left(3-2 e^{\beta\left(\tau_{0}-\tau\right)}\right) \\
M(\tau) & =\frac{2}{9} \beta^{2} R_{0}^{3} e^{2 \beta\left(\tau_{0}-\tau\right)} .
\end{aligned}
$$

Then, we can see that this case is similar to the one for $-1<w<-1 / 3$. In particular, $\dot{R}(\tau)$ has a maximal at $\tau_{\text {min. }}$, where

$$
\begin{aligned}
\dot{R}\left(\tau_{\text {min. }}\right) & =-2^{1 / 3} \beta R_{0} \\
\tau_{\text {min. }} & \equiv \tau_{0}-\frac{1}{\beta} \ln \left(\frac{3}{2}\right) .
\end{aligned}
$$

Thus, by properly choosing the free parameters, the solution can be interpreted as representing the gravitational collapse of a dust cloud in the presence of dark energy, in which the collapse will finally lead to the formation of black holes.

When $w<-1$, the solution is that of Eq.(5.7), which can be written as

$$
\begin{aligned}
a(t) & =a_{0} \frac{\left\{1-\left[\beta(|w|-1)\left(t-t_{0}\right)\right]^{\frac{1}{|w|-1}}\right\}^{2 / 3}}{\left[\beta(|w|-1)\left(t-t_{0}\right)\right]^{\frac{2}{3(|w|-1)}}} \\
& = \begin{cases}0, & t=t_{s}, \\
\infty, & t=t_{0},\end{cases}
\end{aligned}
$$

where

$$
t_{s}=t_{0}+\frac{1}{\beta(|w|-1)} .
$$

From Eq.(15.2) we find that the spacetime is singular at both $t_{0}$ and $t_{s}$, while from Eq.(5.19) we obtain

$$
\begin{aligned}
\dot{R}(\tau) & =-\frac{2}{3} \beta R_{0} \frac{\left[\beta(|w|-1)\left(\tau-\tau_{0}\right)\right]^{\frac{1-3|w|}{3(|w|-1)}}}{\left\{1-\left[\beta(|w|-1)\left(\tau-\tau_{0}\right)\right]^{\frac{1}{|w|-1}}\right\}^{1 / 3}} \\
& = \begin{cases}-\infty, & \tau=\tau_{s}, \\
-B, & \tau=\tau_{\text {min. }}, \\
-\infty, & \tau=\tau_{0},\end{cases}
\end{aligned}
$$

where

$$
\begin{aligned}
B & \equiv-\frac{2}{3} \beta R_{0} \frac{(3|w|)^{|w|}}{(3|w|-1)^{|w|-1 / 3}} \\
\tau_{\text {min. }} & =\tau_{0}+\frac{1}{\beta(|w|-1)}\left(\frac{3|w|-1}{3|w|}\right)^{|w|-1}
\end{aligned}
$$

The curve of $\dot{R}$ is that given in Fig. 6 , but now with $\tau_{0}$ and $\tau_{s}$ being exchanged, as in the present case we have $\tau_{0}<\tau_{s}$. If $B<1$, the solutions can be interpreted as representing the gravitational collapse of a dust cloud in the background of phantoms, starting from a moment $\tau_{i}$, where $\tau_{i}>\tau_{A H}^{-}$. The collapse develops an apparent horizon at $\tau_{A H}^{+}$, whereby a black hole is formed. The total mass of the collapsing star now is given by

$$
M(\tau)=\frac{2}{9} \beta^{2} R_{0}^{3}\left[\beta(|w|-1)\left(\tau-\tau_{0}\right)\right]^{-\frac{2|w|}{|w|-1},}
$$

which is finite and non-zero at $\tau_{s}$, when a spacetime singularity is formed.

Similar to the case where $Q=0$ and $w<-1$, the solutions can also be interpreted as representing a white hole converting itself into a black hole [26].

$$
\text { B. } n=1
$$

In this case, Eq.(15.3) reads

$$
\frac{d y}{\left(1+y^{2}\right)^{m}}=-\beta d t
$$

where $m \equiv-w / 2$. When $m=1 / 2$ or $w=-1$, from Eq. (5.5) we find that $Q=0$. Thus, this is the case studied in the last section. When $m=1$, Eq.(15.24) has the solution,

$$
a(t)=A^{-1 / 3} y^{2 / 3}=a_{0} \tan ^{2 / 3}\left[\beta\left(t_{0}-t\right)\right] .
$$

It can be shown that this case is quite similar to the previous case $n=1 / 2$ and $w<-1$. In particular, the curve of $\dot{R}(\tau)$ is quite similar to that given by Fig. 6 . Therefore, the solution in this case can also be interpreted as representing the gravitational collapse of a dust cloud in the background of phantoms, in which a black hole is finally formed.

\section{CONCLUSIONS}

In this paper we studied the gravitational collapse of a spherically symmetric star with finite radius, which is made of homogeneous and isotropic fluid. When the fluid has only one component with the equation of state $p=$ $w \rho$, we showed explicitly in Sec. III that the collapse always forms black holes for $w>-1 / 3$, including the case of a dust cloud where $w=0$. When $w \leq-1 / 3$ the collapse never forms black holes.

In Sec. IV, we considered the collapse of the fluid that consists of two different components, the dust cloud, $\rho_{D M}$, and the dark energy $p=w \rho$, but assuming that, except for their gravitational interaction, there is no other interaction between them. We found that black holes can still be formed, due to the condensation of the dust cloud. At the beginning of the collapse, the dust cloud may not play an important role. But, as the time increases, it will dominate the collapse, so that a black hole is finally formed. 
To study the effects of the interaction between dust cloud and dark energy, in Sec. V we studied the gravitational collapse by assuming that 27]

$$
\frac{\rho}{\rho_{D M}}=A a^{3 n}
$$

where $A$ and $n$ are arbitrary constants. In this case, the interaction is characterized by [cf. Eq.(5.5)]

$$
Q=-3 A(w+n)\left(\frac{\dot{a}}{a}\right) \frac{a^{3 n} \rho_{t}}{\left(1+A a^{3 n}\right)^{2}} .
$$

By considering several specific models, we found similar conclusions as in the case where the interaction vanishes, that is, black holes can still be formed due to the collapse of the dust cloud in the background of dark energy.

When $w<-1$ (phantoms) some models may also be interpreted as the death of a white hole [26], that is, a white hole evaporates through ejecting material, which will later re-collapse to form a black hole.

Our results obtained in this paper do not seemingly support the speculations that black holes do not exist due to the presence of dark energy. Instead, due to the local condensation of the dust cloud, black holes can still be formed even in the background of dark energy. We believe that this is true not only for a dust cloud but also for other matter fields that satisfy the energy conditions [24].

Certainly, one may argue that a collapsing star that consists of homogeneous and isotropic fluid is a very ideal case, and in more realistic cases the internal region of the collapsing star should be inhomogeneous. However, as argued previously, we believe that the main properties of the present model should remain valid even in more realistic cases.

In addition, in this paper we did not consider the junction of the star to the spacetime outside of it. The main reason is that if apparent horizon develops inside the star, the collapse must form a black hole. Of course, different junctions will result in different masses of black holes and different global structures of the spacetime. The investigations of these problems will be reported in another occasion [19].

\section{Acknowledgments}

RGC was supported by a grant from Chinese Academy of Sciences, grants from NSFC, China (No. 10325525 and No.90403029), and a grant from the Ministry of Science and Technology of China (No. TG1999075401).
[1] A. G. Riess et al. [Supernova Search Team Collaboration], Astron. J. 116, 1009 (1998) arXiv:astro-ph/9805201; S. Perlmutter et al. [Supernova Cosmology Project Collaboration], Astrophys. J. 517, 565 (1999) arXiv:astro-ph/9812133; A. G. Riess et al. [Supernova Search Team Collaboration], Astrophys. J. 607, 665 (2004) arXiv:astro-ph/0402512.

[2] C. L. Bennett et al., Astrophys. J. Suppl. 148, 1 (2003) arXiv:astro-ph/0302207; D. N. Spergel et al. [WMAP Collaboration], Astrophys. J. Suppl. 148, 175 (2003) arXiv:astro-ph/0302209.

[3] M. Tegmark et al. [SDSS Collaboration], Phys. Rev. D 69, 103501 (2004) arXiv:astro-ph/0310723; K. Abazajian et al., arXiv:astro-ph/0410239 K. Abazajian et al. [SDSS Collaboration], Astron. J. 128, 502 (2004) arXiv:astro-ph/0403325. K. Abazajian et al. [SDSS Collaboration], Astron. J. 126, 2081 (2003) arXiv:astro-ph/0305492; E. Hawkins et al., Mon. Not. Roy. Astron. Soc. 346, 78 (2003) arXiv:astro-ph/0212375; L. Verde et al., Mon. Not. Roy. Astron. Soc. 335, 432 (2002) arXiv:astro-ph/0112161.

[4] V. Sahni and A.A. Starobinsky, IJMP. D9, 373 (2000).

[5] S.M. Carroll, Living Rev. Rel. 4, 1 (2001) arXiv:astro-ph/0004075.

[6] P.J.E. Peebles and B. Ratra, Rev. Mod. Phys. 75, 559 (2002) arXiv:astro-ph/0207347.

[7] T. Padmanabhan, Phys. Rep. 380, 235 (2003).

[8] V. Sahni, "Dark Matter and Dark Energy," arXiv:astro-ph/0403324 (2004).

[9] V. Sahni, "Cosmological Surprises from Braneworld models of Dark Energy," arXiv:astro-ph/0502032 (2005).
[10] C. Ma, R.R. Caldwell, P. Bode, and L. Wang, Astrophys. J. 521, L1 (1999).

[11] P.G. Ferreira and M. Joyce, Phys. Rev. Lett. 79, 4740 (1997); D.F. Mota, C. van de Bruck, Astron. Astrophys. 421, 71 (2004).

[12] http://www.nature.com/news/2005/050328/full/050328-8.html http://www.bioon.com/TILS/news/200504/97002.html

[13] E. Babichev, V. Dokuchaev, and Yu. Eroshenko, Phys. Rev. Lett. 93, 021102 (2004); S. Nojiri, S.D. Odintsov, Phys. Rev. D70, 103522 (2004) arXiv:hep-th/0408170.

[14] J.R. Oppenheimer and H. Synyder, Phys. Rev. 55, 455 (1939).

[15] P.S. Joshi, Global Aspects in Gravitation and Cosmology (Clarendon, Oxford, 1993). For more recent reviews, see, e.g., R. Penrose, in Black Holes and Relativistic Stars, edited by R.M. Wald (University of Chicago Press, 1998); A. Krolak, Prog. Theor. Phys. Suppl. 136, 45 (1999); P.S. Joshi, Pramana, 55, 529 (2000), and P.S. Joshi, "Cosmic Censorship: A Current Perspective," gr-qc/0206087 (2002); Gravitational Collapse End States, gr-qc/0412082 (2004), and references therein.

[16] W. Israel, Nuovo Cim. 44B, 1 (1966); ibid., 48B, 463 (1967).

[17] N.O. Santos, Mon. Not. R. Astron. Soc. 216, 403 (1985).

[18] G.C. McVittie, Mon. Not. R. Astron. Soc. 93, 325 (1933).

[19] L. Zhou, R.-G. Cai, and A. Wang, "Black hole formation from collapsing dust cloud in a background of dark energy II: Israel's junction conditions," in preparation.

[20] M. E. Cahill and G. C. McVittie J. Math. Phys., 111382 (1970).

[21] E. Poisson and W. Israel, Phys. Rev. D41 1796 (1990); A. 
Wang, J.F. Villas da Rocha, and N.O. Santos, ibid., D56, 7692 (1997); J.F. Villas da Rocha, A. Wang, and N.O. Santos, Phys. Lett. A255, 213 (1999); S.A. Hayward, Phys. Rev. D70, 104027 (2004); Phys. Rev. Lett. 93, 251101 (2004); "Gravitational radiation from dynamical black holes," arXiv:gr-qc/0505080 (2005), and references therein.

[22] H. Bondi, Nature (London), 186, 535 (1960); H. Bondi, M.G.J. van der Burg, and A.W.K. Metzner, Proc. Roy. Soc. Lond. A269, 21 (1962); R.K. Sach, ibid., A270, 103 (1962); Phys. Rev. 128, 2851 (1962).

[23] This gives the total mass of only the internal part of the star and does not include the part of the thin shell, which usually appears on the surface of the star. In the rest of this paper, the total mass of the star will always refer to the total mass of the internal part of the star.

[24] S.W. Hawking and G.F.R. Ellis, The Large Scale Structure of Spacetime (Cambridge University Press, Cambridge, 1973).

[25] M. Abramowitz and I.A. Stegun, Handbook of Mathematical Functions, (Dover Publications, INC., New York, 1972), pp.555-566.

[26] D. M. Eardley, Phys. Rev. Lett. 33, 442 (1974); S.K. Blau, Phys. Rev. D39, 2901 (1989).

[27] R.-G. Cai and A. Wang, JCAP. 0503, 002 (2005). 